\title{
Nano-Precision Tweezers for Mechanosensitive Proteins and Beyond
}

\author{
Taehyun Yang ${ }^{1}$, Celine Park ${ }^{1}$, Sang-Hyun Rah $^{1, *}$, and Min Ju Shon ${ }^{1,2, *}$
}

\begin{abstract}
${ }^{1}$ Department of Physics, Pohang University of Science and Technology, Pohang 37673, Korea, ${ }^{2}$ School of Interdisciplinary Bioscience and Bioengineering, Pohang University of Science and Technology, Pohang 37673, Korea

*Correspondence: mjshon@postech.ac.kr (MJS); sanghyunrah@postech.ac.kr (SHR)

https://doi.org/10.14348/molcells.2022.2026

www.molcells.org
\end{abstract}

\begin{abstract}
Mechanical forces play pivotal roles in regulating cell shape, function, and fate. Key players that govern the mechanobiological interplay are the mechanosensitive proteins found on cell membranes and in cytoskeleton. Their unique nanomechanics can be interrogated using single-molecule tweezers, which can apply controlled forces to the proteins and simultaneously measure the ensuing structural changes. Breakthroughs in high-resolution tweezers have enabled the routine monitoring of nanometer-scale, millisecond dynamics as a function of force. Undoubtedly, the advancement of structural biology will be further fueled by integrating static atomic-resolution structures and their dynamic changes and interactions observed with the force application techniques. In this minireview, we will introduce the general principles of single-molecule tweezers and their recent applications to the studies of force-bearing proteins, including the synaptic proteins that need to be categorized as mechanosensitive in a broad sense. We anticipate that the impact of nano-precision approaches in mechanobiology research will continue to grow in the future.
\end{abstract}

Keywords: mechanosensitive proteins, single-molecule tweezers, SNARE complex, synapse mechanobiology

\section{INTRODUCTION}

Cells are mechanically active systems. They sense physical stimuli from the outside, transduce the input into biochemical signals, and produce mechanical work to take good moves. In developing these capabilities, evolution has forged nanoscale machineries that operate on the precise mechanics of proteins. Reverse engineering of these molecular contraptions therefore depends on our ability to control and follow the tiny motions of proteins.

An increasing number of proteins are being discovered to employ mechanical forces to perform their functions, enriching the field of mechanobiology (Ingber, 2006; Iskratsch et al., 2014). Due to the remarkable advances in structural biology, the structures of many mechanical proteins have been determined at near-atomic resolution. However, the snapshots provided by X-ray crystallography and cryo-electron microscopy (cryo-EM) are static and lack the dynamic features of conformational changes. Mechanosensitive proteins experience forces in vivo, which, by design, may alter their transient structures and interactions. Therefore, for a complete understanding of how these proteins behave, their force-dependent properties need to be explored at high spatiotemporal resolution, ideally with one molecule at a time.

Single-molecule force techniques such as magnetic tweezers and optical tweezers have proved very powerful in manipulating individual biomolecules (Neuman and Nagy, 2008). Although the methods were historically used to probe slow and large changes in nucleic acids, the current platforms offer nanometer resolution with sub-millisecond temporal precision, which can capture the fast dynamics of proteins to

Received 17 November, 2021; revised 10 January, 2022; accepted 12 January, 2022; published online 31 January, 2022 
the level of several amino acid residues. More importantly, application of piconewton forces in tweezer setups unfolds the hidden, load-dependent behavior of biomolecules. While the short-lived conformations and their subtle changes are not easily accessed with conventional methods, single-molecule tweezers "force" the target molecules into a new thermodynamic equilibrium to observe the force-induced states so that their stabilities can be inferred.

In this minireview, we describe how single-molecule tweezers work and how they have recently been utilized to decipher molecular mechanisms behind mechanosensitivity. We focus on representative examples including cytoskeletal proteins, cell surface receptors, and mechanosensitive ion channels, and then special cases found in neuronal synapses are discussed, which we propose to place in the context of mechanobiology. Overall, we think that high-resolution tweezers are indispensable tools that can motivate exciting research in the novel field of nano-precision mechanobiology.

\section{PRINCIPLES OF SINGLE-MOLECULE TWEEZERS}

The two widely used single-molecule force techniques are optical tweezers and magnetic tweezers (other platforms such as atomic force microscopy [AFM] are not explained here because of space limitations, but other sources are available: Hughes and Dougan, 2016; Krieg et al., 2019; Liu et al., 2015a; Yang et al., 2020). Both methods employ micrometer-sized beads to concurrently manipulate and probe the target molecules (Fig. 1A). In optical tweezers, a single molecule of interest is held usually between two polystyrene beads that are moved horizontally with tightly focused laser beams (Bustamante et al., 2021). Magnetic tweezers in contrast track multiple surface-tethered magnetic beads in parallel, where the beads are placed under a pair of magnets and pulled upward (De Vlaminck and Dekker, 2012). A key difference between the two instruments is the natural mode of operation: optical tweezers typically control the bead position and make force measurements ("position clamp"), whereas in magnetic tweezers the force is set by magnet location and the following changes in the bead position are measured ("force clamp"). Note, however, that optical tweezers can carry out force-clamp experiments by implementing a sophisticated feedback system.

Although the two techniques are largely interchangeable in their applications, optical tweezers have been thought to provide superior resolutions whereas magnetic tweezers have gained popularity due to their simple design and high throughput. However, recent advances of magnetic tweezers in both hardware and software significantly improved their measurement resolution, with the state-of-the-art setups showing an overall comparable performance to optical tweezers (Dulin et al., 2015; Huhle et al., 2015; Lansdorp et al., 2013; Shon et al., 2019). Measurement noise in tweezers depends on many factors including the bead-tether geometry and the applied force, but, as a rule of thumb, modern equipment can reliably detect $\sim 1-\mathrm{nm}$ changes in structure with $\sim 1$-ms time resolution in the piconewton regime.

For the interrogation of proteins, DNA handles are covalently attached to the two amino acid residues of single protein molecules. In this way, small changes in the endto-end extension resulting from conformational changes are faithfully reflected in the bead movement. The effective
A Optical tweezers

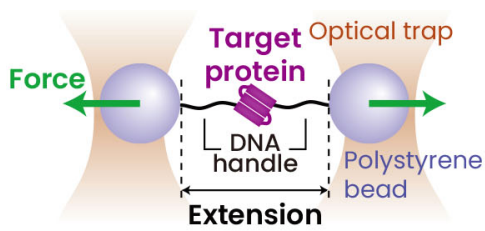

\section{Magnetic tweezers}

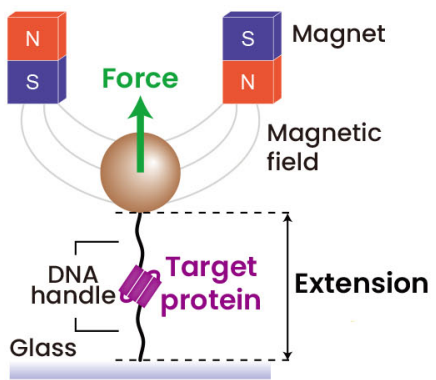

B

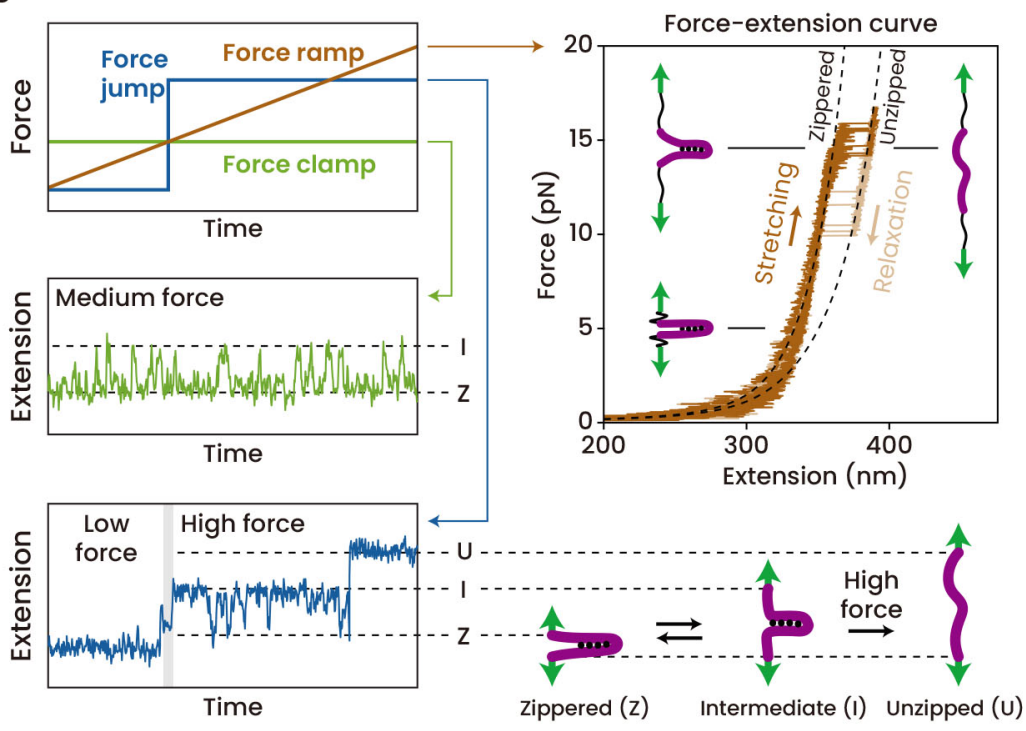

Fig. 1. Principles of single-molecule tweezers. (A) Schematics of optical and magnetic tweezers for the mechanical studies of protein. (B) Types of force application procedure in single-molecule tweezers with hypothetical data expected from a model protein with three observable states (bottom right). At low forces (<10 pN), the protein is zippered (Z). At intermediates forces (10-15 pN), it unfolds to an intermediate structure $(\mathrm{I})$, and at very high forces $(>15 \mathrm{pN})$ it is completely unzippered $(\mathrm{U})$. 
resolutions of tweezers noted above are usually sufficient to probe the structural dynamics at the level of protein domains, sometimes resolving even smaller and faster changes attributed to a few amino-acid residues. One can then vary the applied force to examine the thermodynamics upon mechanical perturbation. Depending on the objective, several types of force-application protocols may be executed (Fig. 1B): (a) a "force ramp" measures the force-extension relation with a gradually changing force; (b) a "force clamp" probes the transition between different states at a fixed force; and (c) a "force jump" induces an abrupt change in the force level to investigate non-equilibrium responses. The three measurements differentially address the elastic properties, intermediate conformations, and transition dynamics and collectively generate a thermodynamic model.

\section{APPLICATIONS TO MECHANOSENSITIVE PROTEINS}

Cellular mechanosensitivity emerges from distinct yet coupled regions: the cytoskeleton and the plasma membrane (Fig. 2A). Cytoskeletal elements respond to global and local mechanical stress, while cell surface receptors and ion channels react to the binding force of ligands and membrane tension. The consequent changes in signal transduction and gene expression often lead to prolonged effects in cell fate and function (Ingber, 2006). Despite the divergent outcomes, common themes in their molecular mechanisms have been identified (Hu et al., 2017; Zhu, 2014). Mechanosensitive proteins change conformations when subjected to forces, and the shape change can recruit other proteins or serve as a substrate for proteolytic cleavage. Some proteins can leverage the force applied to one domain for more pronounced structural rearrangements at distant domains that can perform special functions such as ion channel pore opening (Lin et al., 2019) (Fig. 2B). These effects are reminiscent of allosteric regulation, except that mechanical force functions as a remote effector. Below we will show some examples of this "mechano-allostery" and how single-molecule tweezers have examined their force-bearing properties.

\section{Cytoskeletal proteins}

The high precisions of force application and position measurement in single-molecule tweezers are useful in detecting subtle changes in the force-responsive domains of mechanosensitive proteins. In particular, if the force application points are appropriately selected, the resulting force-induced change can be read as a straightforward measure of mechanosensitivity. The earliest application was in the studies of titin, a giant protein that underlies muscle elasticity (Labeit and Kolmerer, 1995). This filamentous protein with microscale deformations lent itself as an ideal starter for protein-stretching experiments (Kellermayer et al., 1997; Marszalek et al., 1999; Rief et al., 1997). More advanced apparatuses have revealed more details about the titin mechanics (Chen et al., 2015; Pang et al., 2018; Rico et al., 2013; Tapia-Rojo et al., 2019), which is thought to constitute an elaborate mechanosensing scheme (Ibata and Terentjev, 2021; Puchner et al., 2008).

Force-dependent analysis has then been extended toward smaller and more dynamic proteins participating in mechanobiological processes, such as the cytoskeletal protein talin. Talin and its adaptor proteins link actin filaments to the extracellular matrix via focal adhesion complexes (Kanchanawong et al., 2010; Liu et al., 2015b), and this interface then actively mediates mechanical forces (Goult et al., 2018). Single-molecule tweezers have made seminal contributions in elucidating the mechanism of talin function. Most notably, stretching of talin was shown to expose the vinculin binding sites that are usually hidden, thus recruiting vinculin proteins in response to mechanical forces (see Fig. 2B) (del Rio et al., 2009). The following studies have completely characterized the intermediate structures and transitions between them (Wang et al.,
A Cellular mechanosensitivity

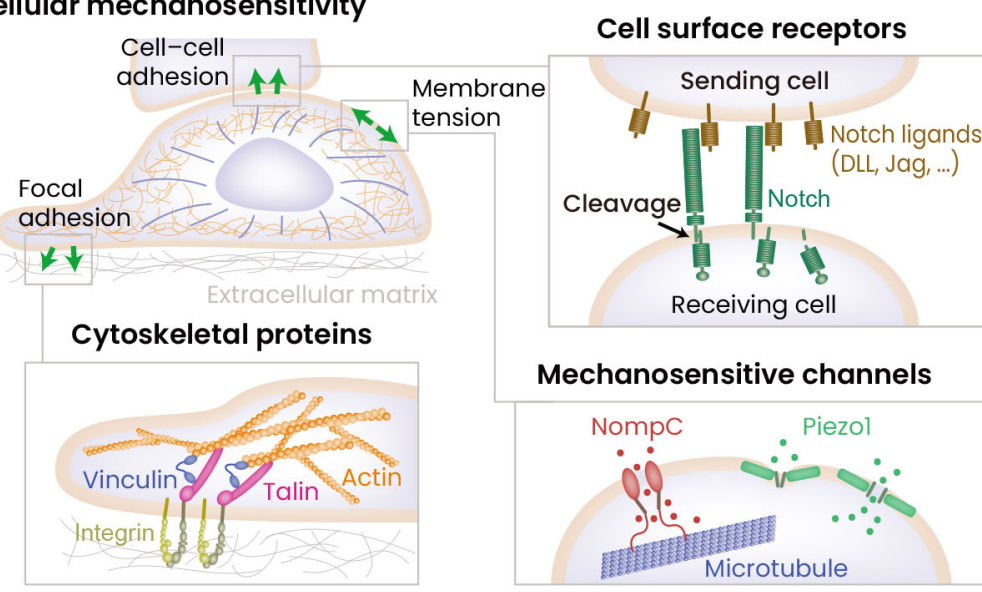

B Mechano-allostery

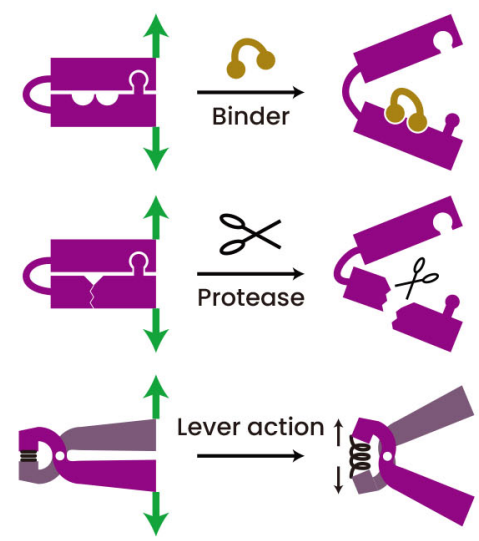

Fig. 2. Application of single-molecule tweezers to mechanosensitive proteins. (A) Cellular landscape of mechanosensitivity. Green arrows indicate the typical directions of forces. Representative examples of mechanosensitive proteins that have been studied with singlemolecule tweezers are shown. (B) Different modes of mechanical allostery in protein conformation change. 
2021; Yao et al., 2016). The piconewton forces used in the in vitro tweezer assays have indeed been shown to be essential for the in vivo functions of talin (Austen et al., 2015; Jiang et al., 2003; Margadant et al., 2011). Similar experiments have been performed on other actin-binding proteins including vinculin (Huang et al., 2017), catenin (Buckley et al., 2014; Yao et al., 2014), filamin (Ferrer et al., 2008; Lee et al., 2009), and actinin (Le et al., 2017; Grison et al., 2017; Roca-Cusachs et al., 2013). All of these studies collectively demonstrate the utility of molecular tweezers in the analysis of cytoskeletal mechanosensitivity.

\section{Cell surface receptors}

Mechanical force has also been found to activate the receptor proteins at cell-cell contact, including the Notch family. Notch proteins are transmembrane receptors critically involved in tissue development and homeostasis (Siebel and Lendahl, 2017), which are activated by binding with ligands (e.g., Delta-like and Jagged families) on the neighboring cell membranes. In their canonical pathway, the ligand binding and subsequent endocytosis had been speculated to generate a pulling force that extends Notch (Parks et al., 2000). This notion was supported by the fact that the protease site in Notch for activation is normally hidden in an autoinhibitory conformation (Gordon et al., 2007). Later, single-molecule tweezers began to probe the forces in Notch-ligand interactions (Meloty-Kapella et al., 2012: Shergill et al., 2012; Stephenson and Avis, 2012), and finally a high-throughput assay has elegantly shown that the proteolytic sensitivity increased when Notch was pulled with small forces exposing the cleavage site (Gordon et al., 2015). Remarkably, the same work showed that piconewton forces were sufficient to activate Notch in live cells. Other studies using force-measuring DNA nanostructures and biomembrane force probe have systematically quantified the force requirement for Notch activation (Wang and Ha, 2013; Luca et al., 2017), further indicating the mechanosensitivity in Notch signaling.

Another classic example of receptor-ligand binding probed with tweezers is the interaction between the von Willebrand factor (VWF) and the platelet receptor glycoprotein Ibo $(\mathrm{GPIb} \alpha)$ that mediates platelet attachment on vessel walls (Ruggeri and Mendolicchio, 2007). The vascular adhesion had long been known to be efficient particularly under high shear conditions such as in the bloodstream, and many questions arise as to the nature of receptor-ligand bonding that initiates the process. Researchers have successfully addressed these problems using the single-molecule force techniques. The earlier works reliably detected the force-dependent interaction between the A1 domain of VWF and GPIb $\alpha$ (Arya et al., 2002; Yago et al., 2008), while the later optical tweezer experiments analyzed the single-molecule kinetics of the same pair with a higher force resolution (Kim et al., 2010; 2015). Interestingly, WWF also exhibits force-induced unfolding and proteolytic cleavage (Zhang et al., 2009), similarly to the above-mentioned Notch receptors. Together, these results have deepened our understanding of how the VWF activity is modulated by the shear flow in the early phase of hemostasis. Other surface receptors that have been studied with single-molecule tweezers include integrins, cadherins, selectins, and T-cell receptors (Chen et al., 2017).

\section{Mechanosensitive channels}

Mechanosensitive channels (MSCs) are transmembrane ion channels gated by mechanical cues (Jin et al., 2020). They are known to play crucial roles in heat and touch sensation, pain, and proprioception. Due to the structural complexity of MSCs, the methodology of measuring extension under bidirectional pulling has not yet been applied to the fulllength proteins. However, several studies have utilized the nano-tweezing capability to study how small forces affect the important domains of MSCs and their physiological behavior.

The ankyrin repeat found in the transient receptor potential (TRP) family is thought to serve as the "gating spring" in hair cell mechanotransduction (Sotomayor et al., 2005). Single-molecule pulling via AFM has measured the elasticity of ankyrin repeats and reported their spring-like behavior (Lee et al., 2006). Later work showed that ankyrin repeats tether NompC (a member of TRP family) to microtubules and are essential to the mechanosensitivity in vivo (Zhang et al., 2015), suggesting a cytoskeleton-dependent gating mechanism. Another example is the Piezo channel, a large, homotrimeric protein with a propeller-shaped structure (Saotome et al., 2018). The three blades of a Piezo channel are profoundly curved and impose a nanodome shape on the residing membrane (Guo and Mackinnon, 2017). Interestingly, the dome structure was found to flatten reversibly when mechanical perturbations were applied by AFM (Lin et al., 2019), which might explain the channel gating by membrane tension (Cox et al., 2016). In another study, the distinct domains of Piezo1 were specifically pulled using magnetic nanoparticles and identified as the domains responsible for mechanosensitivity (Wu et al., 2016).

Nano-tweezers are also useful in controlling MSC behavior by inducing membrane deformations in more complex systems. Optical tweezers were used to tap cell membranes and produce indentations, where the tapping was shown to induce $\mathrm{Ca}^{2+}$ transients in cells expressing Piezo1 (Falleroni et al., 2018). Notably, it was recently discovered that the tension in cell membranes does not propagate over long distances and triggers Piezo channels only in the local vicinity, suggesting localized mechanosensation in subcellular regions (Shi et al., 2018). Finally, tweezing of Piezo1 with magnetic nanoparticles in the brains of freely moving mice can even elicit behavioral changes (Lee et al., 2021), illustrating the specificity and potency of force application approaches.

\section{APPLICATIONS TO SYNAPTIC MECHANOSENSITIVITY}

Although neuronal synapses have not been traditionally seen as mechanosensitive, we argue that the synaptic compartments including the presynapse, the synaptic cleft, and the postsynapse must be viewed from a mechanical perspective (Fig. 3A). More specifically, many neuronal proteins participating in synaptic vesicle trafficking and synaptic remodeling might have evolved to exploit their nanomechanical properties fine-tuned to the synaptic biophysical environment. We summarize below the recent findings from the single-molecule work with high-speed, nano-precision tweezers focusing 

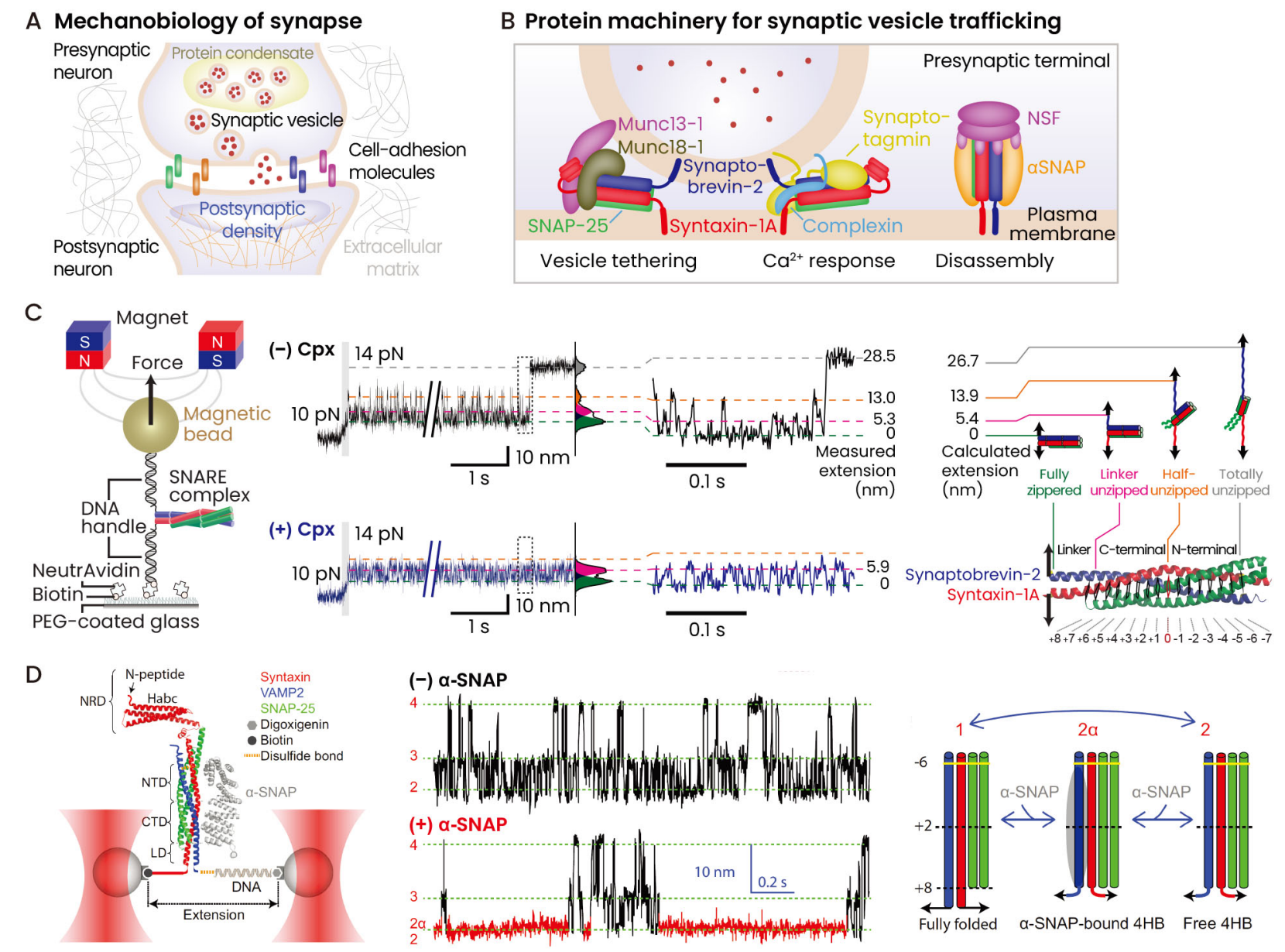

\section{E With $\alpha$ SNAP and NSF}
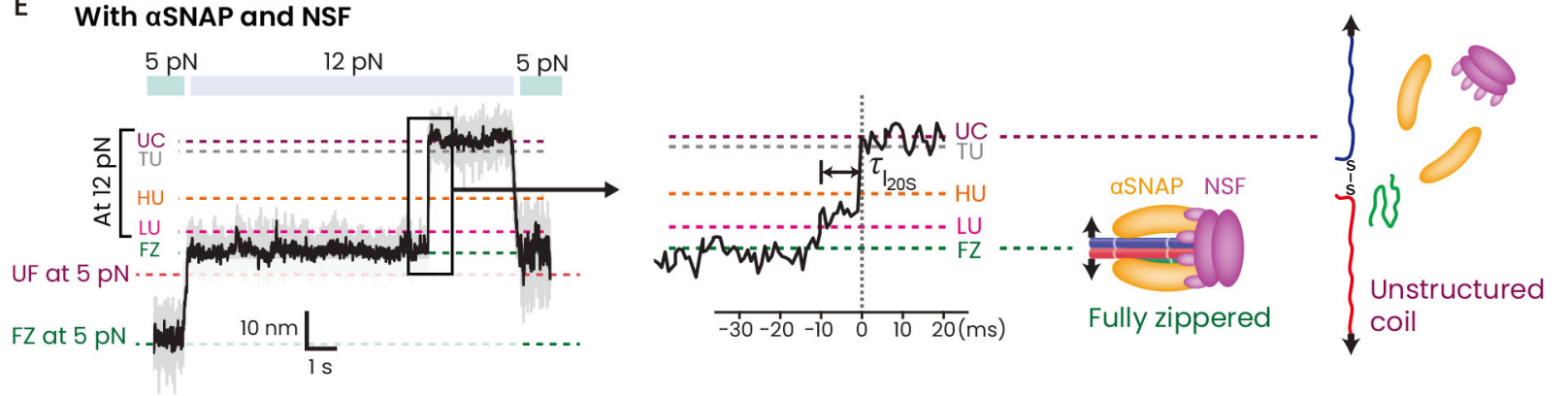

Fig. 3. Application of single-molecule tweezers to synaptic mechanosensitivity. (A) Mechanobiological landscape of a neuronal synapse. (B) Protein machinery for synaptic vesicle trafficking. Representative proteins participating in the different stages of vesicle trafficking are shown. (C) High-resolution magnetic tweezer study of SNARE complex dynamics and the effect of complexin (Cpx). Adapted from the article of Shon et al. (2018) (Nat. Commun. 9, 3639) under Creative Commons Attribution (CC BY 4.0) license. (D) Optical tweezer study on the effect of $\alpha$-SNAP binding to SNARE complex. Adapted from the article of Ma et al. (2016) (Cell Rep. 15, 531-539) under Creative Commons Attribution (CC BY 4.0) license. (E) High-speed observations of $\alpha$-SNAP/NSF-mediated SNARE complex disassembly using magnetic tweezers. Adapted from the article of Kim et al. (2021) (Nat. Commun. 12, 3206) under Creative Commons Attribution (CC BY 4.0) license.

on the presynaptic machinery for vesicle exocytosis (Fig. 3B).

\section{Pre-fusion SNAREs and their regulators}

In evoked neurotransmission, action potential triggers the influx of $\mathrm{Ca}^{2+}$ in presynaptic active zones. Many of the syn- aptic vesicles tethered to the active zone membranes are released via exocytosis within milliseconds upon the arrival of an action potential (Südhof, 2004). To achieve such fast and synchronous release of vesicles in response to the $\mathrm{Ca}^{2+}$ rise, lipid membrane fusion between the vesicle and plasma 
membranes must be tightly controlled in space and time. This is a daunting task given the large energy barrier to the extensive rearrangement of lipid bilayers. The core engine of the neuronal membrane fusion machinery is the complex of three soluble $\mathrm{N}$-ethylmaleimide sensitive factor attachment protein receptors (SNAREs)-synaptobrevin-2 on the vesicle membrane, and syntaxin-1A and SNAP-25 on the plasma membrane (Söllner et al., 1993). These SNARE proteins combine to form an intertwined bundle of $\alpha$-helices (Sutton et al., 1998). It is the binding energy among the SNAREs that drives the membrane fusion and, therefore, requires precise regulation (Yoon and Munson, 2018).

The assembly of SNAREs had been thought to proceed in a directional manner in which the membrane-distal $\mathrm{N}$-termini of syntaxin and synaptobrevin initiate the "zippering" of the complex, thus bringing the vesicle surface in close apposition to the plasma membrane (Pobbati et al., 2006). Experimental attempts to investigate the energetics of SNARE association were initially made using AFM or surface force apparatus (Li et al., 2007; Yersin et al., 2003), but optical and magnetic tweezers with better resolutions have proved more useful in mimicking the zippering and unzipping of the SNARE complex (Gao et al., 2012; Min et al., 2013). By pulling the membrane-proximal ends of syntaxin and synaptobrevin and measuring the small changes in distance between the two ends (Fig. 3C), SNAREs were indeed verified to zipper in the $\mathrm{N}$-to- $\mathrm{C}$ direction. Furthermore, the techniques have shown that SNAREs zipper in multiple discrete steps. The high spatiotemporal resolutions of tweezer data have clearly identified the intermediate states and assigned them to distinct conformations such as "linker-open" and "half-zippered" states (Fig. 3C) (Gao et al., 2012; Shon et al., 2018; Zhang, 2017; Zhang et al., 2016; Zorman et al., 2014). It is important to note that these partially zippered states were observed only under force-loaded conditions, suggesting that the biophysical forces such as the electrostatic repulsion between the opposing membranes might govern the dynamic transition between these intermediates.

Similar mechanical unfolding scheme can interrogate how various binding partners of SNAREs regulate their assembly to enable synchronous neurotransmitter release. A number of proteins interact with SNAREs either directly or indirectly to assist vesicle tethering and form a prefusion complex that allows the immediate response to $\mathrm{Ca}^{2+}$ (Fig. 3B) (Brunger et al., 2018; Südhof, 2013). For example, Munc18 and Munc13 proteins orchestrate the assembly of neuronal SNARE complexes by controlling the conformation of syntaxin (Ma et al., 2013; Zhang and Hughson, 2021). During the tweezing of SNAREs, one can simply add different combinations of such regulatory proteins into the assay buffer, and analyze how the (un)folding of SNARE complexes changes. Results with optical tweezers indeed have shown that Munc18-1 and the MUN domain of Munc13-1 work in concert to template SNAREs into favorable conformations before zippering (Jiao et al., 2018; Kalyana Sundaram et al., 2021; Shu et al., 2020).

Complexin is another regulator of SNAREs and is known for its diverse, seemingly contradictory effects in spontaneous and evoked neurotransmission (Huntwork and Littleton,
2007; Reim et al., 2001; Trimbuch and Rosenmund, 2016). Using high-speed magnetic tweezers, it was found that, in the force-loaded environment, certain domains of complexin stabilize SNARE complexes while preventing their complete zipping toward the membrane-proximal regions (Fig. 3C) (Shon et al., 2018). These results not only agree with previous bulk and single-molecule results from biochemical assays (Choi et al., 2016; Xue et al., 2007; Yoon et al., 2008), but also demonstrate that such force-dependent regulation of SNARE conformation might explain the distinct functions of complexin (Bykhovskaia et al., 2013). It is expected that future studies with high-resolution tweezers on the $\mathrm{Ca}^{2+}$ sensor synaptotagmin and lipid bilayers will fully illuminate the mechanical principles of the membrane fusion machinery (Ma et al., 2017; Zhou et al., 2017).

\section{Post-fusion SNAREs and their regulators}

Following neurotransmitter release, the post-fusion SNARE complexes must be recycled to support additional rounds of vesicle fusion. Disassembling the tightly clustered helices requires a large amount of energy, so the AAA+ ATPase called N-ethylmaleimide sensitive factor (NSF) and its adaptor proteins $\alpha$-SNAPs have evolved together to perform this mechanochemical conversion of energy. $\alpha$-SNAP monomers first bind to a SNARE complex and in turn guide the docking of hexameric NSF proteins. Tweezer experiments have verified the coordinated binding of $\alpha$-SNAP and NSF and their effects on the stability of single SNARE complexes (Fig. 3D) (Kim et al., 2021; Ma et al., 2016; Ryu et al., 2015). Intriguingly, $\alpha$-SNAP also exhibited the dual function of central stabilization and linker destabilization of SNAREs, similarly to the effect of complexin. The concerted action of $\alpha$-SNAP and NSF in the presence of ATP efficiently disassembles the SNARE complex at the force levels far below the mechanical unfolding. In addition, high-speed (1.2-kHz) tweezers proved essential to the detection of short-lived intermediates during the disassembly with a transit time of a few milliseconds (Fig. $3 \mathrm{E})$. Based on these results, the symmetric unfolding model was conceived in which the four SNARE motifs (and consequently all SNARE proteins in the complex) are synchronously unraveled (Kim et al., 2021).

\section{FUTURE PERSPECTIVES}

We have just entered a new era of structural biology with the advent of two powerful tools: single-particle cryo-EM (Cheng, 2018) and DeepMind's AlphaFold2 (Jumper et al., 2021). They will revolutionize the research into protein structures by solving challenging problems at an unprecedented speed. What would be the role of nano-precision tweezers in the coming years? We foresee that their key contributions will be in examining the mechanosensitive assemblies to clarify their molecular mechanisms, shedding light on how these systems function in vivo. In this regard, proteins with unconventional motifs and intrinsically disordered regions might particularly benefit from tweezer experiments that yield a wealth of information on their ever-changing conformations. One intriguing extension would be to introduce phase-separated protein condensates into the assay to investigate their 
mechanoregulatory effects on nearby molecules.

High-resolution tweezers yet remain esoteric because of the craftsmanship associated with sample preparation. We expect that novel methods for the sample synthesis (Kostrz et al., 2019; Maciuba et al., 2021) will make the tweezer experiments more practical and expand the repertoire of neuronal proteins that can be analyzed, such as the weakly interacting molecules in the synaptic cleft and the postsynaptic density. Additionally, correlative measurements in a tweezer setup equipped with fluorescence optics or an electrophysiology rig could enable experiments with primary neurons to gain insight into the mechanobiology of native synapses. Despite the solid progress in live-cell tweezer approaches (Arbore et al., 2019; Mohammed et al., 2019), attempts to collect high-resolution data with living cells will encounter another level of difficulties from various sources of noise. Nevertheless, we believe that this represents an exciting direction that will tackle fundamental unanswered questions in mechanobiology at the single-molecule level.

\section{ACKNOWLEDGMENTS}

This work was supported by the National Research Foundation of Korea (NRF) grant funded by the Korea government (MSIT) (No. 2021R1F1A1056373, No. 2021R1A4A1031754, and No. 2021R1C1C2009717).

\section{AUTHOR CONTRIBUTIONS}

T.Y., C.P., S.H.R., and M.J.S. conducted literature review. T.Y. and S.H.R. prepared the original draft, and M.J.S. wrote the manuscript with inputs from all authors. T.Y., C.P., S.H.R., and M.J.S. prepared the figures.

\section{CONFLICT OF INTEREST}

The authors have no potential conflicts of interest to disclose.

\section{ORCID}

Taehyun Yang

Celine Park

Sang-Hyun Rah

Min Ju Shon

http://orcid.org/0000-0002-1320-4323

http://orcid.org/0000-0002-6072-1139

http://orcid.org/0000-0002-9480-7731

http://orcid.org/0000-0002-0333-1150

\section{REFERENCES}

Arbore, C., Perego, L., Sergides, M., and Capitanio, M. (2019). Probing force in living cells with optical tweezers: from single-molecule mechanics to cell mechanotransduction. Biophys. Rev. 11, 765-782.

Arya, M., Anvari, B., Romo, G.M., Cruz, M.A., Dong, J.F., McIntire, L.V., Moake, J.L., and López, J.A. (2002). Ultralarge multimers of von Willebrand factor form spontaneous high-strength bonds with the platelet glycoprotein Ib-IX complex: studies using optical tweezers. Blood 99, 3971-3977.

Austen, K., Ringer, P., Mehlich, A., Chrostek-Grashoff, A., Kluger, C., Klingner, C., Sabass, B., Zent, R., Rief, M., and Grashoff, C. (2015). Extracellular rigidity sensing by talin isoform-specific mechanical linkages. Nat. Cell Biol. 17, 1597-1606.

Brunger, A.T., Choi, U.B., Lai, Y., Leitz, J., and Zhou, Q. (2018). Molecular mechanisms of fast neurotransmitter release. Annu. Rev. Biophys. 47, 469497.

Buckley, C.D., Tan, J., Anderson, K.L., Hanein, D., Volkmann, N., Weis, W.I., Nelson, W.J., and Dunn, A.R. (2014). Cell adhesion. The minimal cadherin- catenin complex binds to actin filaments under force. Science 346 , 1254211.

Bustamante, C.J., Chemla, Y.R., Liu, S., and Wang, M.D. (2021). Optical tweezers in single-molecule biophysics. Nat. Rev. Methods Primers 1, 25.

Bykhovskaia, M., Jagota, A., Gonzalez, A., Vasin, A., and Littleton, J.T. (2013). Interaction of the complexin accessory helix with the $\mathrm{C}$-terminus of the SNARE complex: molecular-dynamics model of the fusion clamp. Biophys. J. 105, 679-690.

Chen, H., Yuan, G., Winardhi, R.S., Yao, M., Popa, I., Fernandez, J.M., and Yan, J. (2015). Dynamics of equilibrium folding and unfolding transitions of titin immunoglobulin domain under constant forces. J. Am. Chem. Soc. $137,3540-3546$

Chen, Y., Ju, L., Rushdi, M., Ge, C., and Zhu, C. (2017). Receptor-mediated cell mechanosensing. Mol. Biol. Cell 28, 3134-3155.

Cheng, Y. (2018). Single-particle cryo-EM-how did it get here and where will it go. Science $361,876-880$.

Choi, U.B., Zhao, M., Zhang, Y., Lai, Y., and Brunger, A.T. (2016). Complexin induces a conformational change at the membrane-proximal C-terminal end of the SNARE complex. Elife 5, e16886.

Cox, C.D., Bae, C., Ziegler, L., Hartley, S., Nikolova-Krstevski, V., Rohde, P.R. $\mathrm{Ng}$, C.A., Sachs, F., Gottlieb, P.A., and Martinac, B. (2016). Removal of the mechanoprotective influence of the cytoskeleton reveals PIEZO1 is gated by bilayer tension. Nat. Commun. 7, 10366.

De Vlaminck, I. and Dekker, C. (2012). Recent advances in magnetic tweezers. Annu. Rev. Biophys. 41, 453-472.

del Rio, A., Perez-Jimenez, R., Liu, R., Roca-Cusachs, P., Fernandez, J.M., and Sheetz, M.P. (2009). Stretching single talin rod molecules activates vinculin binding. Science 323, 638-641.

Dulin, D., Cui, T.J., Cnossen, J., Docter, M.W., Lipfert, J., and Dekker, N.H. (2015). High spatiotemporal-resolution magnetic tweezers: calibration and applications for DNA dynamics. Biophys. J. 109, 2113-2125.

Falleroni, F., Torre, V., and Cojoc, D. (2018). Cell mechanotransduction with piconewton forces applied by optical tweezers. Front. Cell. Neurosci. 12, 130.

Ferrer, J.M., Lee, H., Chen, J., Pelz, B., Nakamura, F., Kamm, R.D., and Lang, M.J. (2008). Measuring molecular rupture forces between single actin filaments and actin-binding proteins. Proc. Natl. Acad. Sci. U. S. A. 105, 9221-9226.

Gao, Y., Zorman, S., Gundersen, G., Xi, Z., Ma, L., Sirinakis, G., Rothman, J.E., and Zhang, Y. (2012). Single reconstituted neuronal SNARE complexes zipper in three distinct stages. Science 337, 1340-1343.

Gordon, W.R., Vardar-Ulu, D., Histen, G., Sanchez-Irizarry, C., Aster, J.C., and Blacklow, S.C. (2007). Structural basis for autoinhibition of Notch. Nat. Struct. Mol. Biol. 14, 295-300.

Gordon, W.R., Zimmerman, B., He, L., Miles, L.J., Huang, J., Tiyanont, K., McArthur, D.G., Aster, J.C., Perrimon, N., Loparo, J.J., et al. (2015). Mechanical allostery: evidence for a force requirement in the proteolytic activation of Notch. Dev. Cell 33, 729-736.

Goult, B.T., Yan, J., and Schwartz, M.A. (2018). Talin as a mechanosensitive signaling hub. J. Cell Biol. 217, 3776-3784.

Grison, M., Merkel, U., Kostan, J., Djinović-Carugo, K., and Rief, M. (2017). $\alpha$-Actinin/titin interaction: a dynamic and mechanically stable cluster of bonds in the muscle Z-disk. Proc. Natl. Acad. Sci. U. S. A. 114, 1015-1020.

Guo, Y.R. and MacKinnon, R. (2017). Structure-based membrane dome mechanism for Piezo mechanosensitivity. Elife 6, e33660.

Hu, X., Margadant, F.M., Yao, M., and Sheetz, M.P. (2017). Molecular stretching modulates mechanosensing pathways. Protein Sci. 26, 13371351.

Huang, D.L., Bax, N.A., Buckley, C.D., Weis, W.I, and Dunn, A.R. (2017). Vinculin forms a directionally asymmetric catch bond with F-actin. Science 


\section{$357,703-706$}

Hughes, M.L. and Dougan, L. (2016). The physics of pulling polyproteins: a review of single molecule force spectroscopy using the AFM to study protein unfolding. Rep. Prog. Phys. 79, 076601.

Huhle, A., Klaue, D., Brutzer, H., Daldrop, P., Joo, S., Otto, O., Keyser, U.F. and Seidel, R. (2015). Camera-based three-dimensional real-time particle tracking at kHz rates and Ångström accuracy. Nat. Commun. 6, 5885.

Huntwork, S. and Littleton, J.T. (2007). A complexin fusion clamp regulates spontaneous neurotransmitter release and synaptic growth. Nat. Neurosci. 10, 1235-1237.

Ibata, N. and Terentjev, E.M. (2021). Why exercise builds muscles: titin mechanosensing controls skeletal muscle growth under load. Biophys. J. 120, 3649-3663.

Ingber, D.E. (2006). Cellular mechanotransduction: putting all the pieces together again. FASEB J. 20, 811-827.

Iskratsch, T., Wolfenson, H., and Sheetz, M.P. (2014). Appreciating force and shape - the rise of mechanotransduction in cell biology. Nat. Rev. Mol. Cell Biol. 15, 825-833.

Jiang, G., Giannone, G., Critchley, D.R., Fukumoto, E., and Sheetz, M.P. (2003). Two-piconewton slip bond between fibronectin and the cytoskeleton depends on talin. Nature 424, 334-337.

Jiao, J., He, M., Port, S.A., Baker, R.W., Xu, Y., Qu, H., Xiong, Y., Wang, Y., Jin, H., Eisemann, T.J., et al. (2018). Munc18-1 catalyzes neuronal SNARE assembly by templating SNARE association. Elife 7, e41771.

Jin, P., Jan, L.Y., and Jan, Y.N. (2020). Mechanosensitive ion channels: structural features relevant to mechanotransduction mechanisms. Annu. Rev. Neurosci. 43, 207-229.

Jumper, J., Evans, R., Pritzel, A., Green, T., Figurnov, M., Ronneberger, O., Tunyasuvunakool, K., Bates, R., Žídek, A., Potapenko, A., et al. (2021). Highly accurate protein structure prediction with AlphaFold. Nature 596, 583-589.

Kalyana Sundaram, R.V., Jin, H., Li, F., Shu, T., Coleman, J., Yang, J., Pincet, F., Zhang, Y., Rothman, J.E., and Krishnakumar, S.S. (2021). Munc13 binds and recruits SNAP25 to chaperone SNARE complex assembly. FEBS Lett. 595, 297-309.

Kanchanawong, P., Shtengel, G., Pasapera, A.M., Ramko, E.B., Davidson, M.W., Hess, H.F., and Waterman, C.M. (2010). Nanoscale architecture of integrin-based cell adhesions. Nature 468, 580-584.

Kellermayer, M.S.Z., Smith, S.B., Granzier, H.L., and Bustamante, C. (1997). Folding-unfolding transitions in single titin molecules characterized with laser tweezers. Science 276, 1112-1116.

Kim, C., Shon, M.J., Kim, S.H., Eun, G.S., Ryu, J.K., Hyeon, C., Jahn, R., and Yoon, T.Y. (2021). Extreme parsimony in ATP consumption by $20 \mathrm{~S}$ complexes in the global disassembly of single SNARE complexes. Nat. Commun. 12, 3206.

Kim, J., Hudson, N.E., and Springer, T.A. (2015). Force-induced onrate switching and modulation by mutations in gain-of-function von Willebrand diseases. Proc. Natl. Acad. Sci. U. S. A. 112, 4648-4653.

Kim, J., Zhang, C.Z., Zhang, X., and Springer, T.A. (2010). A mechanically stabilized receptor-ligand flex-bond important in the vasculature. Nature 466, 992-995.

Kostrz, D., Wayment-Steele, H.K., Wang, J.L., Follenfant, M., Pande, V.S., Strick, T.R., and Gosse, C. (2019). A modular DNA scaffold to study protein-protein interactions at single-molecule resolution. Nat. Nanotechnol. 14, 988-993.

Krieg, M., Fläschner, G., Alsteens, D., Gaub, B.M., Roos, W.H., Wuite, G.J.L., Gaub, H.E., Gerber, C., Dufrêne, Y.F., and Müller, D.J. (2019). Atomic force microscopy-based mechanobiology. Nat. Rev. Phys. 1, 41-57.

Labeit, S. and Kolmerer, B. (1995). Titins: giant proteins in charge of muscle ultrastructure and elasticity. Science 270, 293-296.
Lansdorp, B.M., Tabrizi, S.J., Dittmore, A., and Saleh, O.A. (2013). A highspeed magnetic tweezer beyond 10,000 frames per second. Rev. Sci. Instrum. 84, 044301.

Le, S., Hu, X., Yao, M., Chen, H., Yu, M., Xu, X., Nakazawa, N., Margadant, F.M., Sheetz, M.P., and Yan, J. (2017). Mechanotransmission and mechanosensing of human alpha-actinin 1. Cell Rep. 21, 2714-2723.

Lee, G., Abdi, K., Jiang, Y., Michaely, P., Bennett, V., and Marszalek, P.E. (2006). Nanospring behaviour of ankyrin repeats. Nature 440, 246-249.

Lee, H., Pelz, B., Ferrer, J.M., Kim, T., Lang, M.J., and Kamm, R.D. (2009). Cytoskeletal deformation at high strains and the role of cross-link unfolding or unbinding. Cell. Mol. Bioeng. 2, 28-38.

Lee, J., Shin, W., Lim, Y., Kim, J., Kim, W.R., Kim, H., Lee, J.H., and Cheon, J. (2021). Non-contact long-range magnetic stimulation of mechanosensitive ion channels in freely moving animals. Nat. Mater. 20, 1029-1036.

Li, F., Pincet, F., Perez, E., Eng, W.S., Melia, T.J., Rothman, J.E., and Tareste, D. (2007). Energetics and dynamics of SNAREpin folding across lipid bilayers. Nat. Struct. Mol. Biol. 14, 890-896.

Lin, Y.C., Guo, Y.R., Miyagi, A., Levring, J., MacKinnon, R., and Scheuring, S. (2019). Force-induced conformational changes in PIEZO1. Nature 573, 230-234.

Liu, B., Chen, W., and Zhu, C. (2015a). Molecular force spectroscopy on cells. Annu. Rev. Phys. Chem. 66, 427-451.

Liu, J., Wang, Y., Goh, W.I., Goh, H., Baird, M.A., Ruehland, S., Teo, S., Bate, N., Critchley, D.R., Davidson, M.W., et al. (2015b). Talin determines the nanoscale architecture of focal adhesions. Proc. Natl. Acad. Sci. U. S. A. 112, E4864-E4873.

Luca, V.C., Kim, B.C., Ge, C., Kakuda, S., Wu, D., Roein-Peikar, M., Haltiwanger, R.S., Zhu, C., Ha, T., and Garcia, K.C. (2017). Notch-Jagged complex structure implicates a catch bond in tuning ligand sensitivity. Science 355, 1320-1324

Ma, C., Su, L., Seven, A.B., Xu, Y., and Rizo, J. (2013). Reconstitution of the vital functions of Munc18 and Munc13 in neurotransmitter release. Science 339, 421-425.

Ma, L., Cai, Y., Li, Y., Jiao, J., Wu, Z., O'Shaughnessy, B., De Camilli, P., Karatekin, E., and Zhang, Y. (2017). Single-molecule force spectroscopy of protein-membrane interactions. Elife 6, e30493.

Ma, L., Kang, Y., Jiao, J., Rebane, A.A., Cha, H.K., Xi, Z., Qu, H., and Zhang, Y. (2016). $\alpha$-SNAP enhances SNARE zippering by stabilizing the SNARE fourhelix bundle. Cell Rep. 15, 531-539.

Maciuba, K., Zhang, F., and Kaiser, C.M. (2021). Facile tethering of stable and unstable proteins for optical tweezers experiments. Biophys. J. 120, 2691-2700.

Margadant, F., Chew, L.L., Hu, X., Yu, H., Bate, N., Zhang, X., and Sheetz, M. (2011). Mechanotransduction in vivo by repeated talin stretch-relaxation events depends upon vinculin. PLoS Biol. 9, e1001223.

Marszalek, P.E., Lu, H., Li, H., Carrion-Vazquez, M., Oberhauser, A.F., Schulten, K., and Fernandez, J.M. (1999). Mechanical unfolding intermediates in titin modules. Nature 402, 100-103.

Meloty-Kapella, L., Shergill, B., Kuon, J., Botvinick, E., and Weinmaster, G. (2012). Notch ligand endocytosis generates mechanical pulling force dependent on dynamin, epsins, and actin. Dev. Cell 22, 1299-1312.

Min, D., Kim, K., Hyeon, C., Cho, Y.H., Shin, Y.K., and Yoon, T.Y. (2013). Mechanical unzipping and rezipping of a single SNARE complex reveals hysteresis as a force-generating mechanism. Nat. Commun. 4, 1705.

Mohammed, D., Versaevel, M., Bruyère, C., Alaimo, L., Luciano, M., Vercruysse, E., Procès, A., and Gabriele, S. (2019). Innovative tools for mechanobiology: unraveling outside-in and inside-out mechanotransduction. Front. Bioeng. Biotechnol. 7, 162.

Neuman, K.C. and Nagy, A. (2008). Single-molecule force spectroscopy: optical tweezers, magnetic tweezers and atomic force microscopy. Nat. 


\section{Methods 5, 491-505.}

Pang, S.M., Le, S., and Yan, J. (2018). Mechanical responses of the mechanosensitive unstructured domains in cardiac titin. Biol. Cell 110, 6576.

Parks, A.L., Klueg, K.M., Stout, J.R., and Muskavitch, M.A. (2000). Ligand endocytosis drives receptor dissociation and activation in the Notch pathway. Development 127, 1373-1385.

Pobbati, A.V., Stein, A., and Fasshauer, D. (2006). N- to C-terminal SNARE complex assembly promotes rapid membrane fusion. Science 313, 673676.

Puchner, E.M., Alexandrovich, A., Kho, A.L., Hensen, U., Schäfer, L.V., Brandmeier, B., Gräter, F., Grubmüller, H., Gaub, H.E., and Gautel, M. (2008). Mechanoenzymatics of titin kinase. Proc. Natl. Acad. Sci. U. S. A. $105,13385-13390$.

Reim, K., Mansour, M., Varoqueaux, F., McMahon, H.T., Südhof, T.C., Brose, $\mathrm{N}$., and Rosenmund, C. (2001). Complexins regulate a late step in $\mathrm{Ca}^{2+}-$ dependent neurotransmitter release. Cell 104, 71-81.

Rico, F., Gonzalez, L., Casuso, I., Puig-Vidal, M., and Scheuring, S. (2013). High-speed force spectroscopy unfolds titin at the velocity of molecular dynamics simulations. Science 342, 741-743.

Rief, M., Gautel, M., Oesterhelt, F., Fernandez, J.M., and Gaub, H.E. (1997). Reversible unfolding of individual titin immunoglobulin domains by AFM. Science 276, 1109-1112.

Roca-Cusachs, P., del Rio, A., Puklin-Faucher, E., Gauthier, N.C., Biais, N. and Sheetz, M.P. (2013). Integrin-dependent force transmission to the extracellular matrix by $\alpha$-actinin triggers adhesion maturation. Proc. Natl. Acad. Sci. U. S. A. 110, E1361-E1370.

Ruggeri, Z.M. and Mendolicchio, G.L. (2007). Adhesion mechanisms in platelet function. Circ. Res. 100, 1673-1685.

Ryu, J.K., Min, D., Rah, S.H., Kim, S.J., Park, Y., Kim, H., Hyeon, C., Kim, H.M., Jahn, R., and Yoon, T.Y. (2015). Spring-loaded unraveling of a single SNARE complex by NSF in one round of ATP turnover. Science 347, 14851489.

Saotome, K., Murthy, S.E., Kefauver, J.M., Whitwam, T., Patapoutian, A. and Ward, A.B. (2018). Structure of the mechanically activated ion channel Piezo1. Nature 554, 481-486.

Shergill, B., Meloty-Kapella, L., Musse, A.A., Weinmaster, G., and Botvinick, E. (2012). Optical tweezers studies on Notch: single-molecule interaction strength is independent of ligand endocytosis. Dev. Cell 22, 1313-1320.

Shi, Z., Graber, Z.T., Baumgart, T., Stone, H.A., and Cohen, A.E. (2018). Cell membranes resist flow. Cell 175, 1769-1779.e13.

Shon, M.J., Kim, H., and Yoon, T.Y. (2018). Focused clamping of a single neuronal SNARE complex by complexin under high mechanical tension. Nat. Commun. 9, 3639.

Shon, M.J., Rah, S.H., and Yoon, T.Y. (2019). Submicrometer elasticity of double-stranded DNA revealed by precision force-extension measurements with magnetic tweezers. Sci. Adv. 5, eaav1697.

Shu, T., Jin, H., Rothman, J.E., and Zhang, Y. (2020). Munc13-1 MUN domain and Munc18-1 cooperatively chaperone SNARE assembly through a tetrameric complex. Proc. Natl. Acad. Sci. U. S. A. 117, 10361041.

Siebel, C. and Lendahl, U. (2017). Notch signaling in development, tissue homeostasis, and disease. Physiol. Rev. 97, 1235-1294.

Söllner, T., Whiteheart, S.W., Brunner, M., Erdjument-Bromage, H., Geromanos, S., Tempst, P., and Rothman, J.E. (1993). SNAP receptors implicated in vesicle targeting and fusion. Nature 362, 318-324.

Sotomayor, M., Corey, D.P., and Schulten, K. (2005). In search of the haircell gating spring: elastic properties of ankyrin and cadherin repeats. Structure 13, 669-682.

Stephenson, N.L. and Avis, J.M. (2012). Direct observation of proteolytic cleavage at the S2 site upon forced unfolding of the Notch negative regulatory region. Proc. Natl. Acad. Sci. U. S. A. 109, E2757-E2765.

Südhof, T.C. (2004). The synaptic vesicle cycle. Annu. Rev. Neurosci. 27, 509-547.

Südhof, T.C. (2013). Neurotransmitter release: the last millisecond in the life of a synaptic vesicle. Neuron $80,675-690$.

Sutton, R.B., Fasshauer, D., Jahn, R., and Brunger, A.T. (1998). Crystal structure of a SNARE complex involved in synaptic exocytosis at $2.4 \AA$ resolution. Nature 395, 347-353.

Tapia-Rojo, R., Eckels, E.C., and Fernández, J.M. (2019). Ephemeral states in protein folding under force captured with a magnetic tweezers design. Proc. Natl. Acad. Sci. U. S. A. 116, 7873-7878.

Trimbuch, T. and Rosenmund, C. (2016). Should I stop or should I go? The role of complexin in neurotransmitter release. Nat. Rev. Neurosci. 17, 118125.

Wang, X. and Ha, T. (2013). Defining single molecular forces required to activate integrin and notch signaling. Science 340, 991-994.

Wang, Y., Yao, M., Baker, K.B., Gough, R.E., Le, S., Goult, B.T., and Yan, J. (2021). Force-dependent interactions between talin and full-length vinculin. J. Am. Chem. Soc. 143, 14726-14737.

Wu, J., Goyal, R., and Grandl, J. (2016). Localized force application reveals mechanically sensitive domains of Piezo1. Nat. Commun. 7, 12939.

Xue, M., Reim, K., Chen, X., Chao, H.T., Deng, H., Rizo, J., Brose, N., and Rosenmund, C. (2007). Distinct domains of complexin I differentially regulate neurotransmitter release. Nat. Struct. Mol. Biol. 14, 949-958.

Yago, T., Lou, J., Wu, T., Yang, J., Miner, J.J., Coburn, L., López, J.A., Cruz, M.A., Dong, J.F., Mclntire, L.V., et al. (2008). Platelet glycoprotein Ib $\alpha$ forms catch bonds with human WT vWF but not with type $2 \mathrm{~B}$ von Willebrand disease vWF. J. Clin. Invest. 118, 3195-3207.

Yang, B., Liu, Z., Liu, H., and Nash, M.A. (2020). Next generation methods for single-molecule force spectroscopy on polyproteins and receptorligand complexes. Front. Mol. Biosci. 7, 85

Yao, M., Goult, B.T., Klapholz, B., Hu, X., Toseland, C.P., Guo, Y., Cong, P., Sheetz, M.P., and Yan, J. (2016). The mechanical response of talin. Nat. Commun. 7, 11966.

Yao, M., Qiu, W., Liu, R., Efremov, A.K., Cong, P., Seddiki, R., Payre, M., Lim, C.T., Ladoux, B., Mège, R.M., et al. (2014). Force-dependent conformational switch of $\alpha$-catenin controls vinculin binding. Nat. Commun. 5, 4525.

Yersin, A., Hirling, H., Steiner, P., Magnin, S., Regazzi, R., Hüni, B., Huguenot, P., Rios, P.D.L., Dietler, G., Catsicas, S., et al. (2003). Interactions between synaptic vesicle fusion proteins explored by atomic force microscopy. Proc. Natl. Acad. Sci. U. S. A. 100, 8736-8741.

Yoon, T.Y., Lu, X., Diao, J., Lee, S.M., Ha, T., and Shin, Y.K. (2008). Complexin and $\mathrm{Ca} 2+$ stimulate SNARE-mediated membrane fusion. Nat. Struct. Mol. Biol. 15, 707-713.

Yoon, T.Y. and Munson, M. (2018). SNARE complex assembly and disassembly. Curr. Biol. 28, R397-R401.

Zhang, W., Cheng, L.E., Kittelmann, M., Li, J., Petkovic, M., Cheng, T., Jin, P., Guo, Z., Göpfert, M.C., Jan, L.Y., et al. (2015). Ankyrin repeats convey force to gate the NOMPC mechanotransduction channel. Cell 162, 1391-1403.

Zhang, X., Halvorsen, K., Zhang, C.Z., Wong, W.P., and Springer, T.A. (2009). Mechanoenzymatic cleavage of the ultralarge vascular protein von Willebrand factor. Science 324, 1330-1334.

Zhang, X., Rebane, A.A., Ma, L., Li, F., Jiao, J., Qu, H., Pincet, F., Rothman, J.E., and Zhang, Y. (2016). Stability, folding dynamics, and long-range conformational transition of the synaptic t-SNARE complex. Proc. Natl. Acad. Sci. U. S. A. 113, E8031-E8040.

Zhang, Y. (2017). Energetics, kinetics, and pathway of SNARE folding and assembly revealed by optical tweezers. Protein Sci. 26, 1252-1265.

Zhang, Y. and Hughson, F.M. (2021). Chaperoning SNARE folding and 
assembly. Annu. Rev. Biochem. 90, 581-603.

Zhou, Q., Zhou, P., Wang, A.L., Wu, D., Zhao, M., Südhof, T.C., and Brunger, A.T. (2017). The primed SNARE-complexin-synaptotagmin complex for neuronal exocytosis. Nature 548, 420-425.

Zhu, C. (2014). Mechanochemitry: a molecular biomechanics view of mechanosensing. Ann. Biomed. Eng. 42, 388-404.

Zorman, S., Rebane, A.A., Ma, L., Yang, G., Molski, M.A., Coleman, J., Pincet, F., Rothman, J.E., and Zhang, Y. (2014). Common intermediates and kinetics, but different energetics, in the assembly of SNARE proteins. Elife 3, e03348. 